\title{
Hypertension Care Cascade at a Large Urban HIV Clinic in Uganda: A Mixed Methods Study Using the Capability, Opportunity, Motivation for Behavior change (COM-B) model
}

\section{Martin Muddu}

Makerere University Joint AIDS Program (MJAP)

Isaac Ssinabulya

Uganda Heart Institute

Simon P. Kigozi

Infectious Disease Research Collaboration

Rebecca Ssennyonjo

Makerere University Joint AIDS Program

Florence Ayebare

Child Health and Development Center, Makerere University

\section{Rodgers Katwesigye}

Makerere University Joint AIDS Program

Mary Mbuliro

Makerere University Joint AIDS Program

\section{Isaac Kimera}

Makerere University Joint AIDS Program

\section{Chris T. Longenecker}

Case Western Reserve University School of Medicine

\section{Moses R. Kamya}

Makerere University College of Health Scinces, Department of Medicine

Jeremy I. Schwartz

Section of General Internal Medicine, Yale School of Medicine

Anne R. Katahoire

Child Health and Development Center, Makerere University

Fred C. Semitala ( $\square$ semitala@gmail.com)

https://orcid.org/0000-0002-0624-7640

\section{Research}


Keywords: Hypertension and HIV Integration, Barriers, Facilitators, Uganda, COM-B

Posted Date: July 30th, 2020

DOI: https://doi.org/10.21203/rs.3.rs-49530/v1

License: (c) (1) This work is licensed under a Creative Commons Attribution 4.0 International License. Read Full License

Version of Record: A version of this preprint was published at Implementation Science Communications on October 20th, 2021. See the published version at https://doi.org/10.1186/s43058-021-00223-9. 


\section{Abstract}

Background: Persons Living with HIV (PLHIV) receiving antiretroviral therapy (ART) have a high prevalence of hypertension (HTN) and increased risk of mortality from cardiovascular diseases. HTN and HIV care integration is recommended in Uganda, though its implementation has lagged. In this study, we sought to analyze the HTN and HIV care cascades and explore barriers and facilitators of HTN/HIV integration within a large HIV clinic in urban Uganda.

Methods: We conducted an explanatory sequential mixed methods study at Mulago ISS clinic in Kampala, Uganda. We determined proportions of patients in HTN and HIV care cascade steps of: Screened, Diagnosed, Initiated on treatment, Retained, and Controlled. Guided by the Capability, Opportunity, Motivation and Behavior (COM-B) model, we then conducted semi-structured interviews and focus group discussions with healthcare providers and hypertensive PLHIV $(n=45)$. We coded the qualitative data deductively and analyzed the data thematically categorizing them as themes that influenced HTN care positively or negatively. These denoted barriers and facilitators, respectively.

Results: Of 15,953 adult PLHIV, 99.1\% were initiated on ART, 89.5\% were retained in care and $98.0 \%$ achieved control (viral suppression) at one year. All 15,953 (100\%) participants were screened for HTN, of whom $24.3 \%$ had HTN. HTN treatment initiation, one-year retention, and control were low at $1.0 \%, 15.4 \%$ and $5.0 \%$, respectively. Barriers and facilitators of HTN/HIV integration appeared in all three COM-B domains. Barriers included low patient knowledge of HTN complications, less priority by patients for HTN treatment compared to ART, sub-optimal provider knowledge of HTN treatment, lack of HTN treatment protocols, inadequate supply of anti-hypertensive medicines and lack of HTN care performance targets. Facilitators included patients' and providers' interest in HTN/HIV integration, patients' interest in PLHIV peers support, providers' knowledge and skills for HTN screening, optimal ART adherence counseling, and availability of automated BP machines.

Conclusion: The prevalence of HTN among PLHIV is high, but the HTN care cascade is sub-optimal in this successful HIV clinic. To close these gaps, models of integrated HTN/HIV care are urgently needed. These findings provide a basis for designing contextually appropriate interventions for HTN/HIV integration in Uganda and other low and middle-income countries.

\section{Contribution To The Literature}

- We utilized the widely used and validated COM-B model to assess determinants of integrated HTN/HIV care in the urban setting

- To our knowledge, this is the first mixed methods study to analyze the hypertension care cascade in an HIV treatment program and explore barriers and facilitators to HTN/HIV integrated care using the COM-B model.

- The barriers and facilitators identified provide a basis for developing stakeholder informed and well contextualized interventions for HTN/HIV integration in Uganda and other LMICs. 


\section{Introduction}

Persons living with HIV (PLHIV) on antiretroviral therapy (ART) have a high prevalence of hypertension (HTN) [1-3]. In Uganda, approximately $1 / 3$ of PLHIV aged $\geq 18$ years have HTN, a leading cause of cardiovascular disease (CVD) [1, 4-11]. HTN accounts for the largest population attributable risk for myocardial infarction and end stage renal disease in PLHIV [12].

To preserve the gains made in HIV care, the treatment cascade must now be extended to include integration of care for HTN within existing HIV services for dual HTN and HIV control [13-17]. A multicenter trial in rural Uganda demonstrated that an integrated HTN/HIV care model that leverages the HIV infrastructure is preferable to vertical programs for both conditions [16].

Although integration of HTN/HIV care is recommended by the World Health Organization (WHO) and adopted by the Uganda Ministry of Health (MoH) HIV treatment guidelines [18, 19], implementation of these guidelines is sub-optimal in Uganda and other LMICs [20-22].

Key barriers to integrated HTN/HIV care include poor access to HTN medications, low provider knowledge of HTN care, inappropriate task shifting, lack of evidence based treatment protocols and weak systems for monitoring and evaluation [21, 23-26].

In this study, we sought to analyze the care cascades for HTN and HIV among adult PLHIV at a large urban HIV clinic in Uganda in order to identify care gaps. In addition, we explored the barriers to and facilitators of integrating HTN and HIV care through qualitative inquiry guided by the Capability, Opportunity, Motivation and Behavior (COM-B) model [27, 28]. We plan to use findings of this study to guide the design of a contextually appropriate strategy to integrate HTN care into HIV clinics in Uganda and other LMICs.

\section{Methods}

\section{Study design}

We utilized an explanatory sequential mixed methods study design. For the quantitative part of the study, we retrospectively collected data to map the HTN care cascade among adult PLHIV at the Mulago immune suppressive syndrome (Mulago-ISS) clinic in order to identify care gaps.

For the qualitative part, we conducted focus group discussions (FGD) and semi-structured in-depth interviews (IDI) with purposively selected hypertensive PLHIV to explore their understanding, beliefs and perceptions regarding HTN/HIV integrated care. In addition, we conducted semi-structured key informant interviews with healthcare providers at the Mulago ISS clinic to understand their perspectives and experiences regarding integrated HTN/HIV care.

We utilized the Capability, Opportunity, Motivation - Behavior (COM-B) model to explore barriers to and facilitators of HTN/HIV integration [27, 28]. We chose the COM-B model, which forms part of the Behavior 
Change Wheel framework, to understand the capabilities, opportunities and motivations for patients and providers with regard to integration of HTN management into HIV care [27]. The central principle for the COM-B is that changing any behavior requires changing capability, opportunity and/or motivation to perform that behavior [28]. Thus, the COM-B model provides a coherent basis for exploring barriers to and facilitators of integrating HTN and HIV care [27]. (Figure 1).

We followed the consolidated criteria for reporting qualitative research (COREQ) guideline in developing this manuscript [29].

\section{Study setting}

We conducted the study at Mulago ISS, the largest HIV clinic in Uganda providing comprehensive HIV services to over 16,500 PLHIV. The clinic is located within Mulago National Referral and Teaching Hospital Complex in Kampala, Uganda's Capital City. HIV related clinical activities include HIV testing and counseling (HTC) and management of antiretroviral therapy (ART). The clinic also implements differentiated ART delivery models where stable PLHIV (good adherence to ART with sustained viral suppression) receive their ART refills in the community. Mulago ISS clinic is owned and operated by the Makerere University Joint AIDS Program (MJAP).

Clinic services are provided by doctors, nurses, clinical officers, HIV counselors, laboratory technicians, pharmacy technicians, records officers as well as PLHIV expert clients.

In line with the national guidelines for HIV care, PLHIV are routinely screened for non-communicable diseases including HTN. If a PLHIV is diagnosed with HTN, the clinician typically prescribes both ART and anti-hypertensive medicines at the same time and gives the client one future appointment for both conditions. Prior to study commencement, Mulago ISS clinic had already achieved universal screening for HTN among all PLHIV during each clinic visit. All available medicines and services at the clinic are provided at no cost to the patients. This study is one of the Learning, Implementation, Networking, Knowledge and Support (LINKS) programs funded by Resolve to Save Lives, a US based NonGovernmental Organization that is supporting countries globally to improve HTN control. The grant recipient and project implementer in Uganda is MJAP. This two-year program started on April $1^{\text {st }}, 2019$. This formative study was conducted to inform the design of an implementation strategy to integrate HTN and HIV care at this clinic.

\section{Study participants and sampling}

\section{Quantitative study}

We included PLHIV who were (1) ${ }^{3} 18$ years old, (2) enrolled into HIV care at the clinic before May $1^{\text {st }}$, 2018, and (3) had at least one clinic visit between July $1^{\text {st }}, 2019$ and January $1^{\text {st }}, 2020$. We chose this group to enable us to review follow up data for at least one year after ART initiation.

\section{Qualitative study}


We purposively selected PLHIV with HTN as a sub-set of participants in the quantitative study and healthcare providers caring for PLHIV who had been in the clinic for at least one year for the interviews. PLHIV with HTN at Mulago ISS clinic were eligible if they had been on ART for at least one year and did not have a cognitive disorder that precluded their active participation in an interview. We approached patients through telephone calls close to their next scheduled clinic appointment.

Eligible healthcare providers were individuals of different cadres who had been providing care to patients at the Mulago ISS clinic for at least two years. We approached healthcare providers face to face. We recruited participants until we achieved data saturation.

\section{Data collection}

\section{Quantitative study}

We extracted retrospective data from the electronic medical records (EMR) for PLHIV. The EMR at Mulago ISS clinic uses the Uganda $\mathrm{MoH}$ open medical records system which captures patient data on HIV care. The clinic EMR was adapted to include additional data elements on HTN and other non-HIV comorbidities. Data on indicators at every stage of the HTN and HIV care cascades were recorded. The data collection tools were developed to obtain information concerning the HTN care cascade at the Mulago ISS clinic and specifically map out the expected outcomes at each cascade step according to national HIV guidelines and WHO recommendations. The steps of Screening, Diagnosis, Treatment initiation, Retention, Monitoring and Control for HTN and HIV care cascades at the Mulago ISS clinic were quantified as adapted from our previous work in Eastern Uganda [25]. Throughout the quantitative and qualitative parts of our study, hypertension was defined as "having a documented blood pressure (BP) $\geq$ $140 / 90 \mathrm{mmHg}$ or documented use of HTN medications or documented history of hypertension" [25]. Each cascade step was described as a proportion of the prior step and reported as follows for HIV and HTN: screened, diagnosed, treated, retained, monitored and controlled.

\section{Qualitative study}

We used semi-structured interview guides based on the three COM-B domains of Capability, Opportunity and Motivation [27, 28]. All interviews had open-ended questions reflecting patients' and healthcare providers' perceptions and perspectives regarding HTN/HIV integration. Prior to data collection, we pretested the interview guides with hypertensive PLHIV and healthcare providers at the Mulago ISS clinic who were not participating in the study. FA, RS and IA shared the objectives of the study with clinic leaders, healthcare providers and patients who were contacted to participate and conducted the FGDs, semi-structured IDIs and KIIs. FA, IA and RS are all trained social scientists with expertise in qualitative research including conducting FGDs and semi-structured interviews. The interviewers established a relationship with the clinic leaders, healthcare providers and patients prior to study commencement, but were not part of the healthcare team at the clinic, thus limiting potential bias. We conducted four FGDs with each group consisting of six to eight hypertensive PLHIV. Each session lasted 60 minutes. FGDs explored PLHIV's experiences of accessing HTN care and related challenges, alternative care seeking and 
recommendations for service improvement. We conducted six semi-structured IDI with hypertensive PLHIV each lasting 30 minutes. The patient semi-structured IDIs explored individual lived experiences and perceptions regarding HTN care in the HIV clinic. In addition, we conducted 13 semi-structured KIls with healthcare providers at the clinic. The semi-structured KIls explored healthcare providers' experience with providing HTN care in the HIV clinic, gaps in HTN care, training needs for staff and recommendations for improvement. All KIls were conducted in English while IDIs and FGDs were conducted in Luganda the local language. All interviews were audio-recorded and transcribed verbatim in Luganda and then translated into English.

\section{Data analysis}

\section{Quantitative study}

We conducted univariate analyses to describe socio-demographic and other characteristics of the cohort. Means and standard deviations (SDs) were used to describe continuous variables, and percentages and frequencies were used for categorical variables. We then stratified the data into two sub-populations: participants that had HIV alone and those that had both HIV and HTN. We compared baseline characteristics of the two subgroups using $\mathrm{X}^{2}$ or Fisher exact tests for categorical characteristics and $\mathrm{t}$ tests for continuous characteristics.

We conducted descriptive analyses of the frequencies and percentages of patients at each previously defined step compared with the preceding step. We then stratified the cascades by HIV (participants with HIV alone) and HIV\& HTN (participants with both HIV and HTN). We analyzed the data using Stata (version 13).

\section{Qualitative study}

A research team with expertise in public health, social science and clinical care (FA, MM and RS) conducted the thematic content analysis. The team coded transcripts using a deductive approach guided by the COM-B as a coding framework. The coding process was guided by consensual qualitative research (CQR) procedures [30]. First, each team member read three transcripts independently and identified preliminary codes. Through a series of meetings, the team agreed on an initial set of codes. To organize and manage the large set of data, all transcripts were coded in Atlas.ti (version 8) software. FA checked all transcripts for accuracy and completeness before they were uploaded into ATLAS t.i V8 software. IK independently coded eight of the transcripts to establish inter-coder reliability (Kappa 0.80). Through more meetings, researchers developed the final codebook. The codes were then categorized into subthemes, and these were mapped onto the COM-B domains and constructs.

Code reports were generated centrally; FA synthesized the findings and summarized them. Themes that negatively influenced HTN treatment in the HIV clinic denoted barriers, and those that positively influenced HTN treatment denoted facilitators (Table 4). 
We extracted specific quotations from the transcripts to illustrate verbatim expressions of matters that appeared important.

\section{Data validation and feedback to study participants}

\section{Results}

\section{Quantitative study}

\section{Characteristics of study participants}

Between July 2019 and January 2020, 15,953 PLHIV were enrolled in the cohort (68\% female and mean (SD) age of 41 (9.7) years).

Overall, the mean baseline CD 4 cell count was $365.3(S D=292.3)$ cells per $\mathrm{mm}^{3}$. Nearly half of all participants, 7,258 (45.9\%), were taking Tenofovir/Lamivudine/Efavirenz (TDF/3TC/EFV) as the initial antiretroviral therapy (ART) regimen. A total of 7,088 (47\%) were overweight or obese (body mass index $>25$; Table 1).

\section{Hypertension Comorbidity}

The prevalence of HTN among PLHIV over 18 years old was $24.3 \%$, (30.7\% among males and $21.5 \%$ among females). Mean baseline systolic and diastolic BP were $119.2(\mathrm{SD}=47.5) \mathrm{mmHg}$ and 77.0 (SD= 12.8) $\mathrm{mmHg}$, respectively. PLHIV over 50 years old had a higher prevalence of HTN at $44.1 \%$ compared to 12.9\% among PLHIV aged 18-30 years. Similarly, HTN was more prevalent among PLHIV on ART for more than 10 years (41\%) compared to those on ART for less than 5 years (20\%). PLHIV whose initial ART regimen contained Zidovudine (AZT) or Nevirapine (NVP) had higher prevalence of hypertension compared to those whose initial ART contained TDF or EFV (Table 2).

\section{HIV Care Cascade}

For the HIV care cascade, nearly all PLHIV 15,803 (99.1\%) were initiated on ART; 14,141 (88.6\%) were retained in care at the study clinic; 13788 (97.5\%) had viral load monitoring at one year; and 13515 (98.0\%) achieved HIV control (viral suppression) (Figure 2).

\section{Hypertension Care Cascade}

Of the 15,953 (100\%) PLHIV who were screened for HTN, 3,892 (24.4\%) were diagnosed with HTN, with only 39 (1.0\%) PLHIV initiated on HTN treatment. Six (15.4\%) patients with both HTN and HIV were retained in care and monitored for HTN at one year and only two (5.0\%) achieved HTN control (Figure 2 and $2 \mathrm{~B})$.

\section{Qualitative study}




\section{Participant Characteristics:}

Characteristics of hypertensive PLHIV $(n=32)$ and healthcare providers $(n=13)$ who participated in the study are summarized in Table 3. Here below we describe their perspectives as mapped on the COM-B model of behavior change [27, 28].

\section{Perspectives of PLHIV regarding integrated hypertension-HIV care}

\section{Capability}

Psychological capability: most PLHIV displayed limited knowledge and understanding of the risk factors and complications of HTN. Additionally, the asymptomatic nature of HTN influenced adherence to HTN medication:

"It seems people take HTN as a condition that is not serious and it is after a person getting diagnosed then they get to know that it is really a dangerous disease. I have witnessed it because I have been with it and I had never screened for it and I am wondering how a tiny lady like me would have HTN." (PLHIVno 2, FGD 3).

"I don't take medicines regularly and consistently, when I feel a bit sick that is when, I will buy a few and swallow for some time." (PLHIV no 4, FGD 1).

Many PLHIV indicated that they needed more knowledge of self-management of HTN including education on lifestyle and drug interactions:

“...you would be telling us the dos and don'ts of dealing with high blood pressure." (PLHIV no 4, FGD 4).

"What I would like to know is if I am to swallow both medications for HTN and HIV at the same time, will it cause any problems?" (PLHIV no 8, FGD 2).

\section{Opportunity}

Physical Opportunity: Patients described lack of money and the high cost as key barriers to accessing HTN medication, and thus some resorted to alternative treatments:

"Money is the main challenge we face in trying to access the HTN drugs." (IDI no 3 with a PLHIV).

"The drugs are expensive, I have to take it on a daily basis, and I cannot buy it all at once. Sometimes I fail to get the money, and then I resort to using tealeaves." (PLHIV 2, FGD 3)

All patients across the FGDs and IDIs expressed a need to access HTN medication at no cost, as is the case for ART. Many patients revealed that they buy a few doses of HTN medicines when they feel unwell or have an emergency. Moreover, most patients were skeptical about the quality and efficacy of HTN medicines they would access in private pharmacies as compared to the HIV clinic where there are trained healthcare providers. 
"I request that someone can get us the medication because a HTN patient has to be taking medicine every day. ... When we buy that medicine, we don't get it in full dosage, we buy a little and take until we feel better... then we just remember taking medicine after getting an emergency." (PLHIV no 6, FGD 2).

"Some pharmacies may not give you the right drugs because not everyone operating the pharmacy knows the drugs. While here (HIV clinic) we know they are giving you from the right place." (IDI no 2 with a PLHIV).

\section{Motivation}

Reflective and automatic motivation: Patients' experiences about HTN screening were generally positive as they reported being screened for HTN at every clinic visit.

"As regards the BP, I express my gratitude to the health workers because when we come at the first desk they take our BP measurements and they give us advice when the BP is high." (PLHIV no 6, FGD 3).

All patients supported the idea of receiving HTN and HIV care together at the same clinic visit. In addition, patients reported that improving access to HTN medicine would improve adherence.

"If the treatment was available here, I wouldn't want to go to any other place because it makes life easier since we will be receiving both our HIV and HTN drugs from the same place. .... In that way, I use the same transport to come here and be able to receive both drugs." (PLHIV no 5, FGD 1).

\section{Automatic motivation:}

There were mixed reactions about patients being supported by peer educators in managing HTN. Most patients welcomed peer support for HTN and HIV treatment, reporting that hearing from a client who has controlled both conditions would give them encouragement:

"It would be good to have someone who reminds you all the time as well as counselling you because you get courage from that person especially if her/his HTN and HIV was initially uncontrolled but this person is now well off." (PLHIV no 6, FGD 4).

Patients who had spent longer time on ART reported that they had the automatic motivation to manage HIV and HTN and may not require peer support. These patients expressed knowledge of their condition, understood the importance of treatment adherence, and were motivated to adhere to HTN treatment.

"... for us, who have spent some time on these drugs (ARVs), we already know what we are supposed to do, we don't need someone reminding us, we can do this ourselves because we know what time we are supposed to swallow these drugs and the importance of adhering." (PLHIV no 3, FGD 1).

\section{Healthcare providers' perspectives on integrated hypertension-HIV care}

\section{Capability}


Physical capability: A majority of healthcare providers reported having the necessary skills to measure blood pressure.

"Screening for HTN is a simple procedure. We need a comfortable chair and a table and a BP screening machine, which should be well maintained. Patients should be calm" (KIl, nurse no 2).

"I think it is quite easy because all of us are taught how to measure BP, how to distinguish between normal and high or low." (KIl, Clinical officer no 1).

Psychological capability: Healthcare providers identified a need to enhance their knowledge of HTN through training, continuing medical education, and consultation with senior practitioners. Participants believed that all cadres of healthcare providers need training. They recommended that lessons learnt in HIV care should be leveraged to improve HTN care.

"I feel that we should not reinvent the wheel so much as we try to handle HTN, .... on the side of HIV, we have achieved above 97\% Viral suppression, so I would think that we should transfer the same efforts to manage HTN. We just need to educate and sensitize the nurses, peers educators, doctors, clinical officers, pharmacists and counsellors about HTN and we should do that to the whole spectrum of cadres." (KII, Medical officer no 1).

"I am sure I can do my best, if we have been managing HIV how can we fail with HTN?" (KII, clinical officer no 2).

"In case we are stuck, we refer to our seniors, who also influence clinical decisions." (KIl, clinical officer no 3).

Healthcare providers mentioned that patients give less priority to HTN medicines compared to ART due to a poor understanding of the dangers of untreated HTN.

"Even when you prescribe for them HTN drugs, they do not take them seriously, as they do for ARVS; they prioritize ARV than HTN drugs. That means the education about the dangers of HTN is not enough." (KII, Medical officer no 1).

Healthcare providers mentioned that ART adherence counselling is always emphasized but that counselling for both ART and HTN medications would enhance psychological capability.

"We shall emphasize counselling because you can give a person medicines but he does not take them. Therefore, we shall not be emphasizing only adherence to ART, we shall also include counselling for HTN medications." (KII, nurse no 3).

\section{Opportunity}

Physical opportunity: A majority of healthcare providers reported that despite having digital BP machines at the clinic, the machines were few and poorly maintained. 
"We use digital BP machines and I think the type is called Omron. I have seen a number of them are old. So, we need better machines and better cuffs" (KII, nurse no 1)

"I could suggest may be we get BP machines onto the doctor's table, in case you are not comfortable with what they took (BP), we can take a second reading" (KIl, medical officer no 1).

All cadres of healthcare providers felt that providing free HTN medication in addition to ART might improve the HTN care cascade. Health care providers reported feeling helpless due to a lack of HTN medications to meet their clients' needs. Moreover, healthcare providers believed that with access to HTN medicines, they would be able to support clients to achieve HTN control.

"The top most thing is medicine, drugs, drugs, drugs. We are doing well regarding ART, even our HIV viral suppression is at $97 \%$.... but for HTN, we have done nothing, we can't buy anti-hypertensive medicines, we just prescribe and encourage our clients to go and buy". (KII, nurse no 1).

"If we could get a consistent supply of HTN medicines, I think our role would be to support these clients to take their medication, and I am sure we would control HTN". (KII, nurse no 3).

Healthcare providers noted the lack of proper HTN care documentation in the medical records made follow-up more difficult. The poor documentation led to frequent changes in HTN treatment, unlike ART where the regimes are well documented.

"Clinicians don't note it in the file that they have prescribed a certain drug. For example, a clinician prescribed amlodipine for patient and it's not noted anywhere in patient files. On the next visit I asked the patient which drug they are taking? He said did not remember since he only took it for one month and threw away the paper. Next month another clinician changed to Nifedipine. So, on every other visit they take a different anti-hypertensive which is really bad". (KII, medical officer no 2).

Healthcare providers reported that they lacked up-to-date guidelines, standard operating procedures (SOPs) and job aides for HTN management. They reported receiving some information on WHO and $\mathrm{MoH}$ guidelines during CMEs; however, they also felt that they would benefit from having these guidelines, SOPs and job aides at their workstations.

"...they promised to print out the new guideline in the management of HTN, that's the job aide we would be using, so we are awaiting the printing. They have not printed out but I think they are going to print out". (KIl, nurse no 3).

Healthcare providers mentioned some social opportunities for HTN treatment including task shifting; empowering HIV peer counsellors to screen and nurses to treat HTN. Healthcare providers suggested that capacity-building activities for HTN care should include peer educators since many patients consult them. In addition, nurses reported less involvement in HTN care compared to doctors. Nurses suggested they could contribute significantly to HTN care if they were empowered to manage HTN as well in task shifting. 
"I told you our peer educators screen for BP and they do it well. Majority of our patients trust their peers better than us; if they don't have the right information, they are going to give misleading information". (KIl, Nurse no 1).

"I think there has been less involvement of nurses compared to doctors in HTN management. So, once we empower and support nurses the better .... They are more close to patients than the doctors". (KII, Nurse no 2).

"If you leave all HTN care to the doctor, they won't have enough time to concentrate on complicated cases." (KIl, Nurse no 3).

\section{Reflective motivation}

Healthcare providers expressed dissatisfaction with their services since they are unable to provide HTN medicines to patients. Providers mentioned they prescribe anti-hypertensive medicines for patients and encourage them to buy from private pharmacies. However, patients do not like being referred.

"One patient wondered, "I have HTN and then you are referring me to another place!" so, they don't want to be referred. If we have both HTN and HIV services here, we shall give them a good service". (KII, clinical officer no 1).

Providers also indicated that there were no monitoring indicators, performance targets and systems of data collection for HTN. All PLHIV attending the clinic were screened for HTN, but screening data were not utilized since comprehensive HTN treatment was not being consistently provided.

"The target is that everyone who comes here has to be screened and this has been achieved but because of lack of medicine, treatment is not available." (KII, clinical officer no 2).

"I don't report anywhere the clients I see who have HTN. We hope to get HTN monitoring indicators and performance targets as the project kicks off, but now we have those for HIV care but not for HTN." (KII, medical officer no 2).

\section{Discussion}

In this explanatory sequential mixed methods study, we compared the HIV and HTN care cascades among PLHIV. The goal was to identify gaps in HTN care within a successful HIV clinic which could be targeted in future interventions. We used qualitative inquiry guided by the COM-B model to understand perceptions and experiences of PLHIV and healthcare providers on integrating HTN care into a large urban HIV program in East Africa [23].

This urban HIV clinic is highly successful in terms of HIV treatment cascade metrics. We established that the HIV care cascade achieved the UNAIDS target of initiating more than $95 \%$ of all PLHIV on ART. In addition, approximately $90 \%$ of all PLHIV initiated on ART were retained at one year while $98 \%$ of all 
PLHIV diagnosed achieved viral suppression [31]. Despite great achievements in HIV care, the HTN care cascade among PLHIV in the same clinic was dismal. Despite routine BP screening for all PLHIV $\geq 18$ years old at each visit and diagnosing nearly a quarter (24.3\%) with HTN, only $1 \%$ of all hypertensive PLHIV were started on treatment for HTN. Retention in HTN care and BP control were consequently suboptimal at $15.4 \%$ and $5 \%$ respectively. This disparity in care for HTN even in a setting of excellent HIV services is problematic in a country like Uganda, where HTN is by far the leading risk factor for cardiovascular disease. In Uganda, for example, high blood pressure contributes 4x higher estimated CVD disability adjusted life years (DALYs) compared to high LDL cholesterol [32]. Poor integration of HTN and HIV care has also been observed in rural clinics of Eastern Uganda and other parts of SSA [25, 26].

Higher prevalence of HTN was associated with older age, male sex, longer duration of ART, and ART containing either in Zidovudine or Nevirapine. These findings are consistent with other studies in SSA [2, $3,33,34]$. The association with ART duration and specific ART drugs may be partly confounded by age $[35,36]$.

Our findings complement growing literature that supports integration of care for NCDs like HTN within existing HIV services by leveraging the huge gains attained by HIV programs [13-17]. Both PLHIV and healthcare providers expressed interest in integrated HTN/HIV care. Evidence from other SSA settings shows that integrated HTN/HIV rather than vertical programs are more cost effective and more likely to achieve HTN control $[17,21,37,38]$.

Most hypertensive PLHIV expressed their desire for HTN/HIV peer educators to provide psychosocial support aimed at improving treatment adherence and retention for both conditions [39-42]. An integrated HTN/HIV program should leverage the existing HIV peer network to improve HTN care among PLHIV [43].

Moreover, healthcare providers and patients have an opportunity to leverage existing ART adherence support, which is routinely offered to all PLHIV. Healthcare providers and patients reaffirmed the benefits of high-quality ART adherence support services at the clinic and added that HTN medication adherence could easily be supported in the same manner. PLHIV were hopeful to achieve greater adherence for both conditions if delivered as an integrated service [44].

All healthcare providers and PLHIV peer educators felt that measuring BP was an easy procedure. In addition, the cascade analysis showed that all PLHIV had their BP measured at least once a year. This clinic was already screening all PLHIV for HTN during each clinic visit even before our study started. BP measurement provides a great opportunity to improve the HTN care cascade since it is the entry point to HTN care $[17,45]$. Shifting the role of BP measurement to HIV peer counselors could lead to even greater efficiency and provide an opportunity for counseling; however, this will require training and simple, standardized protocols.

Generally, PLHIV expressed inadequate knowledge of HTN risk, complications, and self-management, and they prioritized adherence to ART over HTN medicines. Studies in Rwanda and South Africa showed similar findings that hypertensive PLHIV lacked knowledge on risk factors and complications of HTN [17, 
45, 46]. Empowering patients with knowledge may provide an opportunity for better treatment adherence and may facilitate self-care [39].

Some healthcare providers expressed inadequate knowledge of HTN treatment algorithms and drug interactions. Training of healthcare providers on HTN management is required for a robust integrated HTN/HIV program [24, 47]. Moreover, healthcare providers lacked simple treatment protocols for HTN within an HIV clinic. HTN/HIV integrated programs need to leverage the WHO HEARTS technical packages for HTN control which provide simple and stepwise evidence based treatment protocols for HTN in the primary healthcare setting [48-52].

Poor access to HTN medications and BP machines have been cited as key barriers to HTN control in SSA [23]. In our study, it was evident from patients and healthcare providers that this inevitably contributed to the poor HTN cascade at Mulago ISS. Currently, patients are asked to pay for HTN medicines out of pocket, but cannot afford them [53]. Simply providing access to effective medicines for HTN at little or no cost may greatly improve HTN cascade metrics and complement the expanded access to ART.

\section{Limitations Of The Study}

This study was conducted at a single, unique HIV clinic; however, Mulago ISS is the largest HIV clinic in Uganda providing ART to over 16,500 PLHIV. HIV services at the clinic are provided in line with the consolidated guidelines for the prevention and treatment of HIV and AIDS in Uganda [19]. In addition, the clinic serves clients from all parts of the country since it is located at and linked to the National Referral Hospital. Given the breadth of the study population, we believe that the findings of this study may be generalizable to other LMIC settings.

\section{Conclusion}

The prevalence of HTN among PLHIV at a large, urban HIV clinic is high but the cascade of care for HTN is poor. Findings from this study provide a basis for designing contextually appropriate and targeted interventions for HTN/HIV integration to close these gaps in Uganda and other LMICs. Key stakeholders should be engaged to identify appropriate interventions. Future research should determine clinical outcomes, cost and cost effectiveness of integrated HTN/HIV services [15, 54].

\section{List Of Abbreviations}

COM-B Capability, Opportunity, Motivation and Behavior

HTN Hypertension

PLHIV Persons Living with HIV

CVD Cardiovascular Disease 
NCD Non-Communicable Disease

ART Antiretroviral Therapy

WHO World Health Organization

MoH Ministry of Health.

FGD Focus group discussion

KII Key Informant Interview

IDI In-depth interview

LMICs Low-and Middle Income Countries

\section{Declarations}

\section{Ethics approval and consent to participate}

This study was approved by the TASO Institutional Review Board (IRB) number TASOREC/040/19-UGREC-009 and the Uganda National Council for Science and Technology (UNCST) number HS 2644. For the quantitative portion of the study, written permission to access the HIV clinic and medical records was obtained from the clinic manager at Mulago ISS. Written informed consent was obtained from each participant in the qualitative portion of the study after explaining the purpose of the study, benefits, approximate time of interview and an assurance of confidentiality of the study results. In case a participant was not willing to be audio recorded for key informant or in-depth interviews, interview notes were taken. Privacy was maintained during data collection, analysis and storage. All identifiable information was removed from final records after data collection to ensure participant anonymity and only the core research team and PIs had access to the de-identified data.

\section{Consent for publication}

Not applicable

\section{Availability of data and materials}

The datasets used and/or analyzed during the current study are available from the corresponding author on reasonable request.

\section{Competing interests}

The authors declare that they have no relevant competing interests

\section{Funding}


Research reported in this manuscript was supported by Resolve to Save Lives under Award Number MJAP/VITAL 2019/001. Dr. Martin Muddu was also supported by the Fogarty International Center of the NIH under Award Number D43 TW010037. The content is solely the responsibility of the authors and does not necessarily represent the official views of Resolve to Save Lives or the NIH. The funders had no role in the study design, data collection, analysis or interpretation. Martin Muddu and Fred C. Semitala had full access to all the data and had the final responsibility for the decision to submit the manuscript for publication.

\section{Author contributions}

MM, FCS, IS, AK, SPK, JIS and IK were responsible for the design of the study and interpretation of data. MMB, RK, RS, IK, RS, IA and RN led data collection and interpretation. FA, SPK, MM and MMB performed data analysis. MRK and CTL participated in the interpretation of data. All authors participated in writing the initial draft of the manuscript. Martin Muddu and Fred. C Semitala participated in writing the final manuscript. All authors read and approved the final manuscript before submission.

\section{Acknowledgement}

We are grateful to the following persons for their invaluable support: The clinic manager and staff of Mulago ISS clinic, and all the research assistants who participated in this study. Srish K. Sharma contributed to designing figures of this manuscript.

\section{References}

1. Mateen FJ, Kanters S, Kalyesubula R, Mukasa B, Kawuma E. Kengne APet al. Hypertension prevalence and Framingham risk score stratification in a large HIV-positive cohort in Uganda. J Hypertens. 2013 Jul;31(7):1372-8. discussion 1378.

2. Alana T, Brennan L, Jamieson NJ, Crowther MP, Fox, Jaya A, George, Kaitlyn M, Berry, et al. Prevalence, incidence, predictors, treatment, and control of hypertension among HIV-positive adults on antiretroviral treatment in public sector treatment programs in South Africa. PLoS One. 2018 Oct;3(10):e0204020. 13(.

3. Alana T, Brennan L, Jamieson NJ, Crowther MP, Fox, Jaya A, George, Kaitlyn M, Berry, et al. Prevalence, incidence, predictors, treatment, and control of hypertension among HIV-positive adults on antiretroviral treatment in public sector treatment programs in South Africa. PLoS One. 2018 Oct;3(10):e0204020. 13(.

4. Gakidou E, Afshin A, Abajobir AA, Abate KH, Abbafati C, Abbas KM, et al. Global, regional, and national comparative risk assessment of 84 behavioural, environmental and occupational, and metabolic risks or clusters of risks, 1990-2016: a systematic analysis for the Global Burden of Disease Study 2016. Lancet. 2017 Sep 16;390(10100):1345-1422.

5. Gakidou E, Afshin A, Abajobir AA, Abate KH, Abbafati C, Abbas KM, et al. Global, regional, and national comparative risk assessment of 84 behavioural, environmental and occupational, and 
metabolic risks or clusters of risks, 1990-2016: a systematic analysis for the Global Burden of Disease Study 2016. Lancet. 2017 Sep 16;390(10100):1345-1422.

6. Lunyera J, Kirenga B, Stanifer JW, Kasozi S, van der Molen T. Katagira Wet al. Geographic differences in the prevalence of hypertension in Uganda: Results of a national epidemiological study. PLoS One. 2018 Aug 1; 13(8):e0201001.

7. Rabkin M, Palma A, McNairy ML, Gachuhi AB, Simelane S, Nuwagaba-Biribonwoha $H$, et al. Integrating cardiovascular disease risk factor screening into HIV services in Swaziland: lessons from an implementation science study. AIDS. 2018 Jul 1; 32 Suppl 1:S43-S46.

8. Patel P, Rose CE, Collins PY, Nuche-Berenguer B, Sahasrabuddhe VV, Peprah E, et al. Noncommunicable diseases among HIV-infected persons in low-income and middle-income countries: a systematic review and meta-analysis. AIDS. 2018 Jul;1(32 Suppl 1):5-20.

9. Kwarisiima D, Balzer L, Heller D, Kotwani P, Chamie G, Clark T, et al. Population-based assessment of hypertension epidemiology and risk factors among HIV-positive and general populations in rural Uganda. PLoS One. 2016 May 27; 11(5):e0156309.

10. Kazooba P, Kasamba I, Mayanja BN, Lutaakome J, Namakoola I, Salome T, et al. Cardiometabolic risk among HIV-POSITIVE Ugandan adults: prevalence, predictors and effect of long-term antiretroviral therapy. Pan Afr Med J. 2017 May;15:27:40.

11. Kalyesubula R, Kayongo A, Semitala FC, Muhanguzi A, Katantazi N, Ayers D, et al. Trends and level of control of hypertension among adults attending an ambulatory HIV clinic in Kampala, Uganda: a retrospective study. BMJ Glob Health. 2016 Nov 24; 1(3):e000055.

12. Keri N, Althoff KA, Gebo RD, Moore CM, Boyd AC, Justice C, Wong, et al. Contributions of traditional and HIV-related risk factors on non-AIDS-defining cancer, myocardial infarction, and end-stage liver and renal diseases in adults with HIV in the USA and Canada: a collaboration of cohort studies. Lancet HIV. 2019 Feb;6(2):e93-104.

13. Pangmekeh PJ, Awolu MM, Gustave S, Gladys T, Cumber SN, et al. Association between highly active antiretroviral therapy (HAART) and hypertension in persons living with HIV/AIDS at the Bamenda regional hospital, Cameroon. Pan Afr Med J. 2019 Jun;6:33:87.

14. Lelani Coetzee LB, De Neve J-W, Bärnighausen T, Geldsetzer P, Sebastian Vollmer. HIV, antiretroviral therapy and non-communicable diseases in sub-Saharan Africa: empirical evidence from 44 countries over the period 2000 to 2016. J Int AIDS Soc. 2019 Jul;22(7):e25364.

15. Osetinsky B, Hontelez JAC, Lurie MN, McGarvey ST, Bloomfield GS, Pastakia SD, et al. Epidemiological And Health Systems Implications Of Evolving HIV And Hypertension In South Africa And Kenya. Health Aff (Millwood). 2019 Jul;38(7):1173-81.

16. Kwarisiima D, Atukunda M, Owaraganise A, Chamie G, Clark T, Kabami J, et al. Hypertension control in integrated HIV and chronic disease clinics in Uganda in the SEARCH study. BMC Public Health. 2019 May 6; 19(1):511.

17. Muyunda Mutemwa N, Peer A, de Villiers B, Mukasa TE, Matsha, Edward J, Mills, et al. Prevalence, detection, treatment, and control of hypertension in human immunodeficiency virus (HIV)-infected 
patients attending HIV clinics in the Western Cape Province, South Africa. Medicine (Baltimore). 2018 Aug; 97(35):e12121.

18. World Health Organization (WHO). March 2014 supplement to the 2013 consolidated guidelines on the use of antiretroviral drugs for treating and preventing HIV infection: recommendations for a public health approach. Available at: http://www.who.int/ hiv/pub/guidelines/arv2013 /arvs2013upplement_march2014/en/ (accessed June 06, 2020).

19. Uganda Ministry of Health. Consolidated Guidelines for Prevention and Treatment of HIV in Uganda. 2018. Available at: http://library.health.go.ug /publications/service-delivery-diseases-controlprevention-communicable-diseases/hivaids/consolidated (accessed June 30, 2020).

20. Walsh KF, Lee MH, Martelly S, Pierre MM, Joseph J, Gustin M, et al. Integrating hypertension services at an HIV clinic in Port-au-Prince, Haiti: A report from the field. J Clin Hypertens (Greenwich). 2018 Oct;20(10):1485-92.

21. Sarah Gimbel AO, Mocumbi K, Ásbjörnsdóttir J, Coutinho L, Andela B, Cebola, et al. Systems analysis and improvement approach to optimize the hypertension diagnosis and case cascade for PLHIV individuals (SAIA-HTN): a hybrid type III cluster randomized trial. Implement Sci. 2020 Mar 6;15(1):15.

22. David W, Dowdy, Kimberly A, Powers, Timothy B. Hallett.Towards evidence-based integration of services for HIV, non-communicable diseases and substance use: insights from modelling. J Int AIDS Soc. 2020 Jun;23(Suppl 1):e25525. Suppl 1.

23. Iwelunmor J, Ezechi O, Obiezu-Umeh C, Gbajabiamila T, Musa AZ, Oladele D. at al. Capabilities, opportunities and motivations for integrating evidence-based strategy for hypertension control into HIV clinics in Southwest Nigeria. PLoS One. 2019 Jun 6; 14(6):e0217703.

24. Tiny Masupe Y, Mashalla E, Seloilwe, Harun Jibril and Heluf Medhin. Integrated management of HIV/NCDs: knowledge, attitudes, and practices of health care workers in Gaborone, Botswana. Afr Health Sci. 2019 Sep;19(3):2312-23.

25. Muddu M, Tusubira AK, Sharma SK, Akiteng AR, Ssinabulya I, Schwartz JI. Integrated Hypertension and HIV Care Cascades in an HIV Treatment Program in Eastern Uganda: a retrospective cohort study. J Acquir Immune Defic Syndr. 2019 Aug;15(5):552-61. 81 (.

26. Muddu M, Tusubira AK, Nakirya B, Nalwoga R, Semitala FC, Akiteng AR, et al. Exploring barriers and facilitators to integrated hypertension-HIV management in Ugandan HIV clinics using the Consolidated Framework for Implementation Research (CFIR). Implementation Science Communications. 2020;1(1):1-14.

27. Susan Michie, Maartje M van, Stralen andRobert West. The behavior change wheel: a new method for characterising and designing behaviour change interventions. Implement Sci. 2011 Apr;23:6:42.

28. Fiona, Barker. Lou Atkins and Simon de Lusignan. Applying the COM-B behaviour model and behaviour change wheel to develop an intervention to improve hearing-aid use in adult auditory rehabilitation. Int J Audiol. 2016 Jul;55(Suppl 3):90-8. 
29. Tong A, Sainsbury P, Craig J. Consolidated criteria for reporting qualitative research (COREQ): a $32-$ item checklist for interviews and focus groups. Int J Qual Health Care. 2007 Dec;19(6):349-57.

30. Depner RM, Grant PC, Byrwa DJ, Breier JM, Lodi-Smith J, Kerr CW, et al. A consensual qualitative research analysis of the experience of inmate hospice caregivers: Posttraumatic growth while incarcerated. Death Stud. 2017 Apr;41(4):199-210.

31. Joint United Nations Programme on HIV/AIDS (UNAIDS). 90-90-90 - An ambitious treatment target to help end the AIDS epidemic. January 2017: Available at: http://www.unaids.org/en/ resources/documents/2017/90-90-90 (accessed June 29, 2020).

32. Daniel Dicker G, Nguyen D, Abate KH, Abate, Solomon M, Abay C. Abbafati et al. Global, regional, and national age-sex-specific mortality and life expectancy, 1950-2017: a systematic analysis for the Global Burden of Disease Study 2017. Lancet. 2018 Nov;10(10159):1684-735. 392(.

33. Noelle A, Benzekri M, Seydi IN, Doye M, Toure MP, Sy, Nancy B, Kiviat, et al. Increasing prevalence of hypertension among HIV-positive and negative adults in Senegal, West Africa, 1994-2015. PLoS One. 2018 Dec;31(12):e0208635. 13(.

34. Temesgen Fiseha AG, Belete. Henok Dereje and Abebe Dires. Hypertension in HIV-Infected Patients Receiving Antiretroviral Therapy in Northeast Ethiopia. Int J Hypertens. 2019 Dec 23;2019:4103604.

35. Victoria Haldane H, Legido-Quigley FLehHChuah, Sigfrid L, Murphy G. Suan Ee Onget al. Integrating cardiovascular diseases, hypertension, and diabetes with HIV services: a systematic review. AIDS Care. 2018 Jan;30(1):103-15.

36. Magafu MG, Moji K, Igumbor EU, Magafu NS, Mwandri M, Mwita JC, et al. Non-communicable diseases in antiretroviral therapy recipients in Kagera Tanzania: a cross-sectional study. Pan Afr Med J. 2013 Nov;6:16:84.

37. Parastu Kasaie B, Weir M, Schnure C, Dun J, Pennington Yu. Teng et al. Integrated screening and treatment services for HIV, hypertension and diabetes in Kenya: assessing the epidemiological impact and cost-effectiveness from a national and regional perspective. J Int AIDS Soc. 2020 Jun;23(Suppl 1(Suppl 1):e25499.

38. Sando David K, Alexander O, Samson KP, Chris G, David M, Gerald M, Winnie, et al. Cost-effectiveness analysis of integrating screening and treatment of selected non-communicable disease risk factors into HIV/AIDS treatment in Uganda. J Int AIDS Soc. 2020;23:2.

39. Sharon Tsui, Julie A, Denison CE, Kennedy LW, Chang O, Koole K, Torpey, et al. Identifying models of HIV care and treatment service delivery in Tanzania, Uganda, and Zambia using cluster analysis and Delphi survey. BMC Health Serv Res. 2017 Dec 6;17(1):811.

40. Fred C, Semitala CS, Camlin J, Wallenta L, Kampiire RK, Amanyire G, et al. Understanding uptake of an intervention to accelerate antiretroviral therapy initiation in Uganda via qualitative inquiry. $\mathrm{J}$ Int AIDS Soc. 2017 Dec;20(4):e25033.

41. Katuramu Richard K, Moses R, Sanyu, Naome, Mari Armstrong-Hough and Semitala Fred C. Sustainability of the streamlined ART (START-ART) implementation intervention strategy among ART- 
eligible adult patients in HIV clinics in public health centers in Uganda: a mixed methods study. Implementation Science Communications, 2020. 1: p. 1-12.

42. Carol S, Camlin ED, Charlebois E, Geng F, Semitala J, Wallenta M, Getahun, et al. Redemption of the "spoiled identity:" the role of HIV-positive individuals in HIV care cascade interventions. J Int AIDS Soc. 2017 Dec;20(4):e25023.

43. Ojo TT, Hawley NL, Desai MM, Akiteng AR. David Guwatudde and Jeremy I Schwartz. Exploring knowledge and attitudes toward non-communicable diseases among village health teams in Eastern Uganda: a cross-sectional study. BMC Public Health. 2017 Dec 12;17(1):947.

44. Siedner JManne-Goehler,MJ, Montana L, Harling G, Geldsetzer P, Rohr J, et al. Hypertension and diabetes control along the HIV care cascade in rural South Africa. J Int AIDS Soc. 2019 Mar;22(3):e25213.

45. George S, McGrath N, Oni T. The association between a detectable HIV viral load and noncommunicable diseases comorbidity in HIV positive adults on antiretroviral therapy in Western Cape, South Africa. BMC Infect Dis. 2019 Apr 27;19(1):348.

46. Juvenal, Biraguma. Eugene Mutimura and José M Frantz Knowledge about modifiable risk factors for non-communicable diseases adults living with HIV in Rwanda. Afr Health Sci. 2019 Dec;19(4):3181-9.

47. Aifah Angela ON, Lance R, Cynthia R, Schexnayder Julie B, Gerald B, Hayden, et al. Use of a humancentered design approach to adapt a nurse-led cardiovascular disease prevention intervention in HIV clinics. Progress in Cardiovascular Diseases, 2020.

48. Vedanthan R, Bernabe-Ortiz A, Herasme Ol, et al. Innovative approaches to hypertension control in low-and middle-income countries. Cardiol Clin. 2017;35:99-115.

49. Rabkin M, de Pinho H, Michaels-Strasser S, et al. Strengthening the health workforce to support integration of HIV and noncommunicable disease services in sub-Saharan Africa. AIDS. 2018;32:4754.

50. World health Organization (WHO). Technical package for cardiovascular disease management in primary health care: team-based care. Available at: https://creativecommons.org/licenses/by-ncsa/3.0/igo) (accessed June 26,2020).

51. World Health Organization (WHO). Technical package for cardiovascular disease management in primary health care: evidence-based treatment protocols. Available at: https://creativecommons.org/ licenses/by-nc-sa/3.0/igo) (accessed June 26,2020).

52. World health Organization (WHO). Technical package for cardiovascular disease management in primary health care: systems for monitoring. Available at: https://creativecommons.org/licenses/bync-sa/3.0/igo). (accessed June 26,2020).

53. Sonak D, Pastakia DN, Tran I, Manji C, Wells. Kyle Kinderknecht and Robert Ferris. Building reliable supply chains for noncommunicable disease commodities: lessons learned from HIV and evidence needs. AIDS. 2018 Jul 1;32 Suppl 1:S55-S61. 
54. Anupam Garrib J, Birungi S, Lesikari I, Namakoola TN, Cuevas L, et al. Integrated care for human immunodeficiency virus, diabetes and hypertension in Africa. Trans R Soc Trop Med Hyg. 2019 Dec 1;113(12):809-812.

\section{Tables}

Table 1. Baseline characteristics of the study participants 


\begin{tabular}{|c|c|c|c|c|}
\hline Characteristic & $\begin{array}{l}\text { Overall Cohort } \\
(n=15,953)\end{array}$ & $\operatorname{HIV}(n=12,061)$ & $\begin{array}{l}\text { HIV \& HTN } \\
(n=3,892)\end{array}$ & $\begin{array}{l}P \\
\text { value }\end{array}$ \\
\hline Age, yrs & $40.7(S D=9.7)$ & $39.4(\mathrm{SD}=9.2)$ & $44.7(S D=10.4)$ & $<0.001$ \\
\hline \multicolumn{4}{|l|}{ Age categories, yrs } & $<0.001$ \\
\hline 18 to 30 & $1,983(12.4 \%)$ & 1,728 (14.3\%) & 255 (6.6\%) & \\
\hline 31 to 50 & $11,458(71.8 \%)$ & 8,892 (73.7\%) & $2,566(65.9 \%)$ & \\
\hline Over 50 & $2,411(15.1 \%)$ & 1,349 (11.2\%) & $1,062(27.3 \%)$ & \\
\hline \multicolumn{4}{|l|}{ Sex } & $<0.001$ \\
\hline Male & $5,077(31.8 \%)$ & $3,521(29.2 \%)$ & $1,556(40.0 \%)$ & \\
\hline Female & $10,876(68.2 \%)$ & $8,540(70.8 \%)$ & $2,336(60.0 \%)$ & \\
\hline \multicolumn{5}{|l|}{ Baseline BP, mm Hg } \\
\hline Systolic & $119.2(\mathrm{SD}=47.5)$ & $\begin{array}{l}115.5(\mathrm{SD}= \\
49.3)\end{array}$ & $145.7(\mathrm{SD}=15.9)$ & $<0.001$ \\
\hline Diastolic & $77.0(S D=12.8)$ & $74.7(S D=11.4)$ & $93.8(\mathrm{SD}=9.9)$ & $<0.001$ \\
\hline \multicolumn{4}{|l|}{ Baseline ART regimen } & $<0.001$ \\
\hline TDF-3TC-EFV & $7,258(45.9 \%)$ & $5,918(49.7 \%)$ & $1,340(34.5 \%)$ & \\
\hline AZT-3TC-NVP & $4,227(26.7 \%)$ & $2,929(24.6 \%)$ & 1,298 (33.4\%) & \\
\hline AZT-3TC-EFV & $1,801(11.4 \%)$ & $1,306(11.0 \%)$ & 495 (12.7\%) & \\
\hline TDF-3TC-NVP & $1,054(6.7 \%)$ & $752(6.3 \%)$ & $302(7.8 \%)$ & \\
\hline Other & $1,470(9.3 \%)$ & $1,015(8.5 \%)$ & $455(11.7 \%)$ & \\
\hline \multicolumn{4}{|l|}{ Duration on ART } & $<0.001$ \\
\hline$<2$ years & $3,638(23.0 \%)$ & $2,885(24.2 \%)$ & $753(19.4 \%)$ & - \\
\hline 2 to $5 y$ rs & $5,512(34.9 \%)$ & 4,453 (37.4\%) & $1,059(27.2 \%)$ & - \\
\hline 5 to $10 y r s$ & $5,675(35.9 \%)$ & 4,004 (33.6\%) & $1,671(43.0 \%)$ & - \\
\hline$>10 y r s$ & 985 (6.2\%) & $578(4.9 \%)$ & 407 (10.5\%) & - \\
\hline Baseline CD 4 count & $365.3(S D=292.3)$ & $\begin{array}{l}365.9(\mathrm{SD}= \\
292.1)\end{array}$ & $\begin{array}{l}363.6(\mathrm{SD}= \\
293.2)\end{array}$ & 0.674 \\
\hline \multicolumn{4}{|c|}{ Baseline CD 4 count by category } & 0.162 \\
\hline$<50$ & $2,110(13.2 \%)$ & $1,610(13.4 \%)$ & $500(12.9 \%)$ & \\
\hline $50-<100$ & $1,041(6.5 \%)$ & $760(6.3 \%)$ & $281(7.2 \%)$ & \\
\hline $100-<200$ & $2,123(13.3 \%)$ & $1,591(13.2 \%)$ & $532(13.7 \%)$ & \\
\hline
\end{tabular}




\begin{tabular}{|c|c|c|c|c|}
\hline$>200$ & $10,657(66.9 \%)$ & $8,080(67.1 \%)$ & 2,577 (66.3\%) & \\
\hline Baseline BMI, & & & & 0.130 \\
\hline Underweight $(<19.0)$ & $1,221(8.1 \%)$ & $887(7.8 \%)$ & $334(9.0 \%)$ & - \\
\hline $\begin{array}{l}\text { Normal weight (19.0 to } \\
<25.0 \text { ) }\end{array}$ & $6,774(44.9 \%)$ & $5,122(45.0 \%)$ & $1,652(44.6 \%)$ & - \\
\hline $\begin{array}{l}\text { Overweight (25.0 to } \\
<30.0)\end{array}$ & $4,175(27.7)$ & 3,159 (27.8\%) & 1,016 (27.4\%) & - \\
\hline Obese $(>=30.0)$ & $2,913(19.3)$ & 2,210 (19.4\%) & $703(19.0 \%)$ & \\
\hline
\end{tabular}

Table 2: Prevalence of hypertension among PLHIV at the Mulago ISS clinic

\begin{tabular}{|c|c|c|c|}
\hline Patient category & Sub-category & Prevalence (\%) & $P$ value \\
\hline Overall & Overall & $3,892 / 15,953(24.4 \%)$ & - \\
\hline \multirow[t]{2}{*}{ Sex } & Male & $1,556 / 5,077(30.7 \%)$ & \multirow[t]{2}{*}{$<0.001$} \\
\hline & Female & $2,336 / 10,876(21.5 \%)$ & \\
\hline \multirow[t]{3}{*}{ Age category } & $18-30$ & $255 / 1,983(12.9 \%)$ & \multirow[t]{3}{*}{$<0.001$} \\
\hline & $31-50$ & $2,566 / 11,458(22.4 \%)$ & \\
\hline & Over 50 & $1,062 / 2,411(44.1 \%)$ & \\
\hline \multirow[t]{5}{*}{ Baseline ART regimen } & TDF-3TC-EFV & $1,340 / 7,258(18.5 \%)$ & \multirow[t]{5}{*}{$<0.001$} \\
\hline & AZT-3TC-EFV & $495 / 1,801(27.5 \%)$ & \\
\hline & TDF-3TC-NVP & $302 / 1,054(28.7 \%)$ & \\
\hline & AZT-3TC-NVP & $1,298 / 4,227(30.7 \%)$ & \\
\hline & Other & $455 / 1,470$ (31.0\%) & \\
\hline \multirow[t]{4}{*}{ Duration of ART } & $<2$ years & 753/3,638 (20.7\%) & \multirow[t]{4}{*}{$<0.001$} \\
\hline & 2 to 5 years & $1,059 / 5,512(19.2 \%)$ & \\
\hline & 5 to 10 years & $1,671 / 5,675(29.4 \%)$ & \\
\hline & $>10$ years & 407/985 (41.3\%) & \\
\hline
\end{tabular}


Table 3: Number and characteristics of participants for the qualitative study

\begin{tabular}{|c|c|c|c|c|}
\hline Data collection methods & $\begin{array}{l}\text { Number and category of } \\
\text { participants }\end{array}$ & $\begin{array}{l}\text { Total } \\
\text { participants }\end{array}$ & $\begin{array}{l}\text { Female } \\
\mathrm{N}(\%)\end{array}$ & $\begin{array}{l}\text { Mean } \\
\text { age } \\
\text { (SD) }\end{array}$ \\
\hline $\begin{array}{l}\text { Focus Group Discussion (FGDs) } \\
\text { for patients }\end{array}$ & $\begin{array}{l}\text { Patients who had both HTN } \\
\text { and HIV (4 FGDs) }\end{array}$ & 26 & $\begin{array}{l}18 \\
(69.0 \%)\end{array}$ & $\begin{array}{l}52.0 \\
( \pm \\
9.5)\end{array}$ \\
\hline $\begin{array}{l}\text { In-depth Interviews (IDIs) for } \\
\text { patients }\end{array}$ & $\begin{array}{l}\text { Patients who had both HTN } \\
\text { and HIV (6 IDIs) }\end{array}$ & 6 & $\begin{array}{l}4 \\
(66.7 \%)\end{array}$ & $\begin{array}{l}44.0 \\
( \pm \\
9.8)\end{array}$ \\
\hline \multirow[t]{6}{*}{$\begin{array}{l}\text { Key Informant Interviews (KIIs) for } \\
\text { health care providers }\end{array}$} & Doctor & 2 & $\begin{array}{l}1 \\
(50.0 \%)\end{array}$ & N/A \\
\hline & Nurse & 3 & $\begin{array}{l}2 \\
(66.7 \%)\end{array}$ & N/A \\
\hline & Clinical officer & 3 & $\begin{array}{l}2 \\
(66.7 \%)\end{array}$ & $\mathrm{N} / \mathrm{A}$ \\
\hline & Pharmacy technician & 2 & $\begin{array}{l}1 \\
(50.0 \%)\end{array}$ & $\mathrm{N} / \mathrm{A}$ \\
\hline & HIV/HTN Counselor & 2 & $\begin{array}{l}1 \\
(50.0 \%)\end{array}$ & $\mathrm{N} / \mathrm{A}$ \\
\hline & HIV/HTN peer educator & 1 & $\begin{array}{l}1 \\
(100 \%)\end{array}$ & N/A \\
\hline Total number of participants & & 45 & $\begin{array}{l}30 \\
(66.7 \%)\end{array}$ & \\
\hline
\end{tabular}

Table 4. Barriers and facilitators for integrated HTN/HIV care in the HIV clinic as related to the domains of COM-B model 


\begin{tabular}{|c|c|c|}
\hline $\begin{array}{l}\text { COM-B } \\
\text { Domain }\end{array}$ & Barriers & Facilitators \\
\hline \multirow[t]{3}{*}{$\begin{array}{l}\text { Psychological } \\
\text { capability }\end{array}$} & $\begin{array}{l}\text { Patient lack of knowledge } \\
\text { of HTN risk, complications } \\
\text { and self-management }\end{array}$ & $\begin{array}{l}\text { Healthcare providers have adequate knowledge of } \\
\text { HTN screening }\end{array}$ \\
\hline & $\begin{array}{l}\text { Healthcare providers and } \\
\text { patients lack knowledge of } \\
\text { HTN-HIV drug interactions }\end{array}$ & \multirow{2}{*}{$\begin{array}{l}\text { Healthcare providers and patients can leverage ART } \\
\text { adherence support which is already being provided } \\
\text { to PLHIV to provide adherence support for both HTN } \\
\text { and HIV treatment }\end{array}$} \\
\hline & $\begin{array}{l}\text { Lack of monitoring } \\
\text { indicators for HTN }\end{array}$ & \\
\hline \multirow[t]{2}{*}{$\begin{array}{l}\text { Physical } \\
\text { capability }\end{array}$} & \multirow[t]{2}{*}{$\begin{array}{l}\text { There were no barriers in } \\
\text { this domain }\end{array}$} & $\begin{array}{l}\text { HTN/HIV peer educators and healthcare providers } \\
\text { have adequate skills to screen for HTN among } \\
\text { PLHIV }\end{array}$ \\
\hline & & $\begin{array}{l}\text { Measuring BP is easy for most of the healthcare } \\
\text { providers including HIV peer educators }\end{array}$ \\
\hline \multirow[t]{5}{*}{$\begin{array}{l}\text { Physical } \\
\text { opportunity }\end{array}$} & $\begin{array}{l}\text { Lack of simple evidence } \\
\text { based treatment protocol } \\
\text { for HTN care }\end{array}$ & \multirow[t]{5}{*}{$\begin{array}{l}\text { Availability of BP machines and staff to measure } \\
\text { and record blood pressure }\end{array}$} \\
\hline & $\begin{array}{l}\text { Lack of on-site HTN } \\
\text { medications despite } \\
\text { demand from patients and } \\
\text { providers }\end{array}$ & \\
\hline & $\begin{array}{l}\text { Cost of buying anti- } \\
\text { hypertensive medicines is } \\
\text { high; patients can't afford }\end{array}$ & \\
\hline & $\begin{array}{l}\text { Inadequate maintenance of } \\
\text { automated BP machines at } \\
\text { the HIV clinic }\end{array}$ & \\
\hline & $\begin{array}{l}\text { Lack of data collection } \\
\text { tools and databases for } \\
\text { HTN care }\end{array}$ & \\
\hline $\begin{array}{l}\text { Social } \\
\text { opportunity }\end{array}$ & $\begin{array}{l}\text { HTN prescriptions are } \\
\text { mainly done by doctors; } \\
\text { limited task shifting to } \\
\text { clinical officers and nurses. }\end{array}$ & $\begin{array}{l}\text { Patients are interested in being supported by PLHIV } \\
\text { peer educators to improve adherence to HTN and } \\
\text { HIV treatment }\end{array}$ \\
\hline $\begin{array}{l}\text { Reflective } \\
\text { motivation }\end{array}$ & $\begin{array}{l}\text { Patients prioritize } \\
\text { adherence to ART over HTN } \\
\text { medications }\end{array}$ & $\begin{array}{l}\text { Patients and healthcare providers have great interest } \\
\text { in HTN/HIV integrated care }\end{array}$ \\
\hline $\begin{array}{l}\text { Automatic } \\
\text { motivation }\end{array}$ & $\begin{array}{l}\text { Lack of performance } \\
\text { targets and review of HTN } \\
\text { care quality }\end{array}$ & There were no facilitators in this domain \\
\hline
\end{tabular}




\section{Figures}

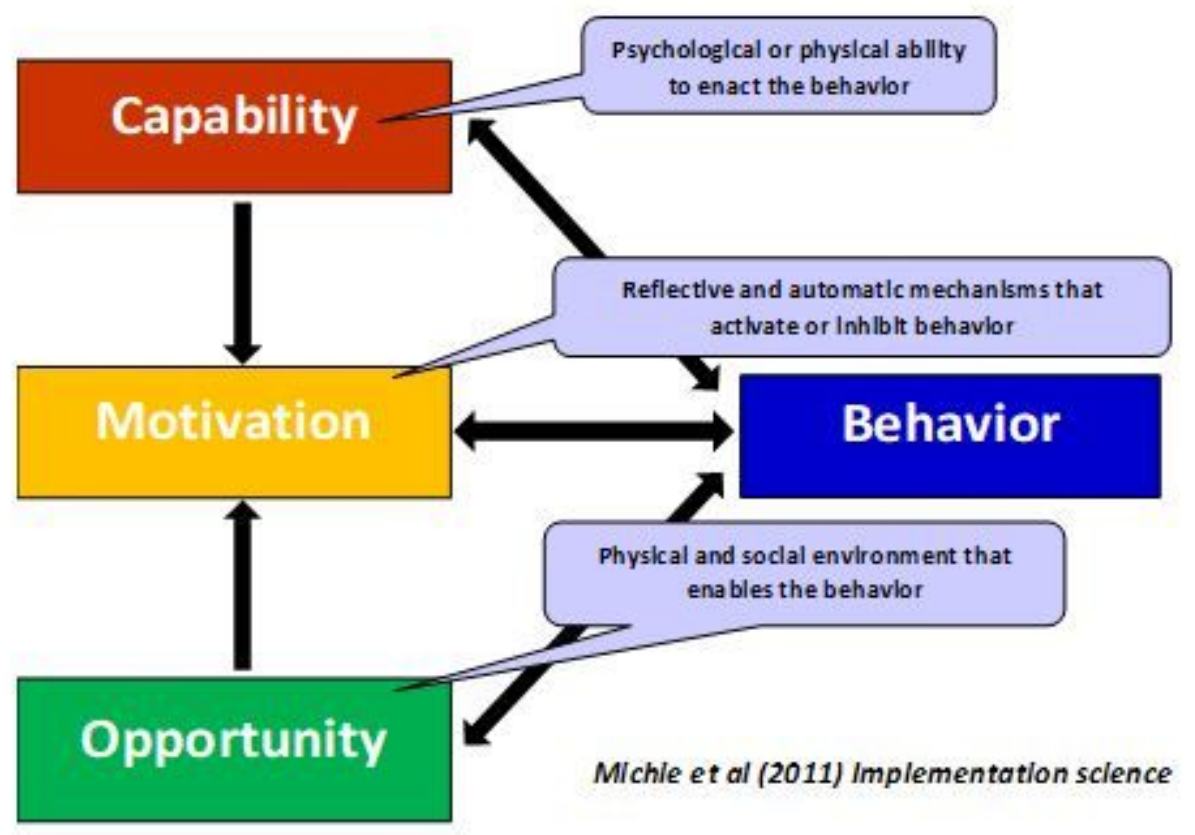

\section{Figure 1}

the Capaility Opportunity Motivation influences Behavior (COM-B) model: adapted from Michie et al [27].

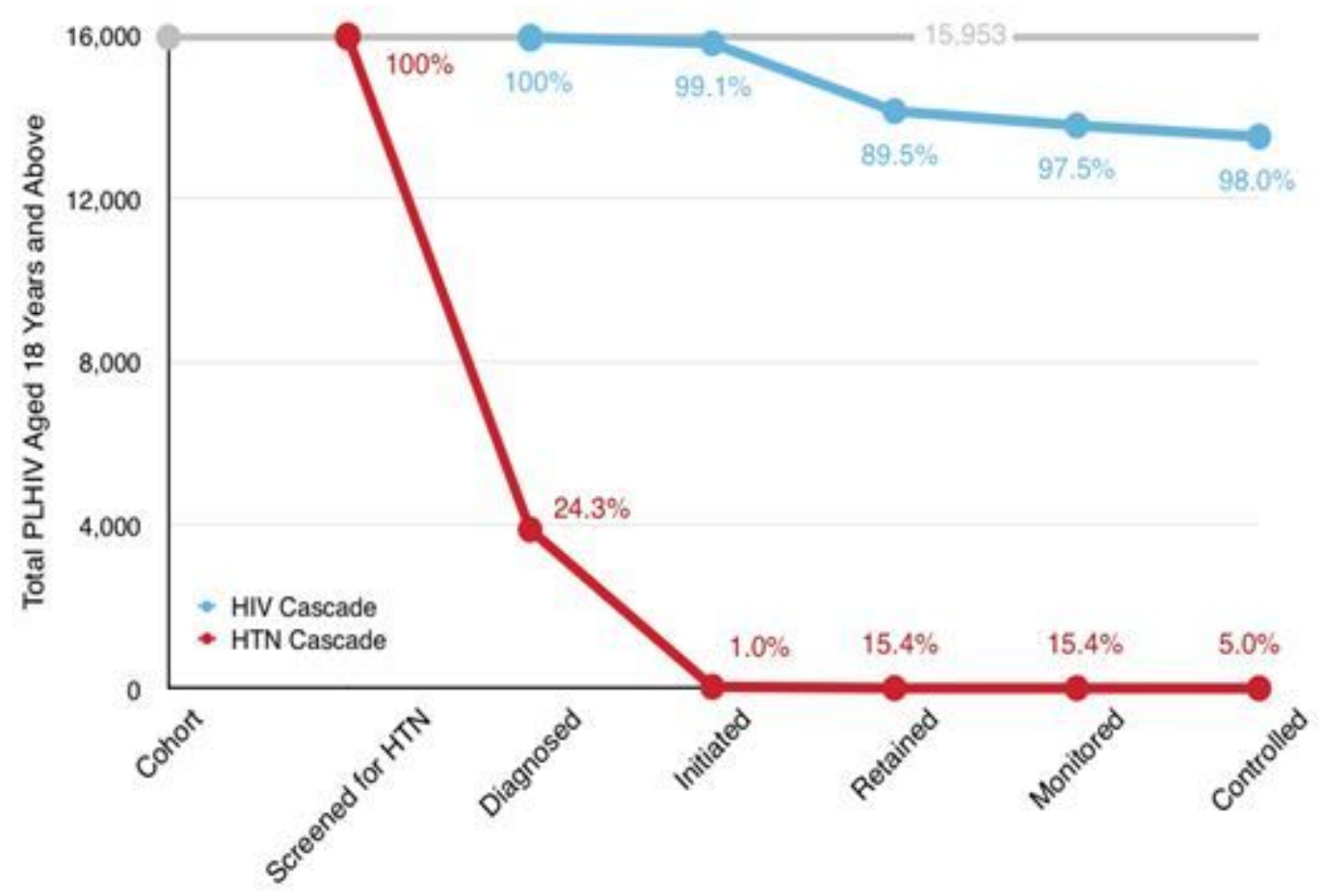


Figure 2

Integrated hypertesion-HIV care cascades at the Mulago ISS clinic The proportions are the percentages of participants at each particular step of the cascade as compared to the previous cascade steps.

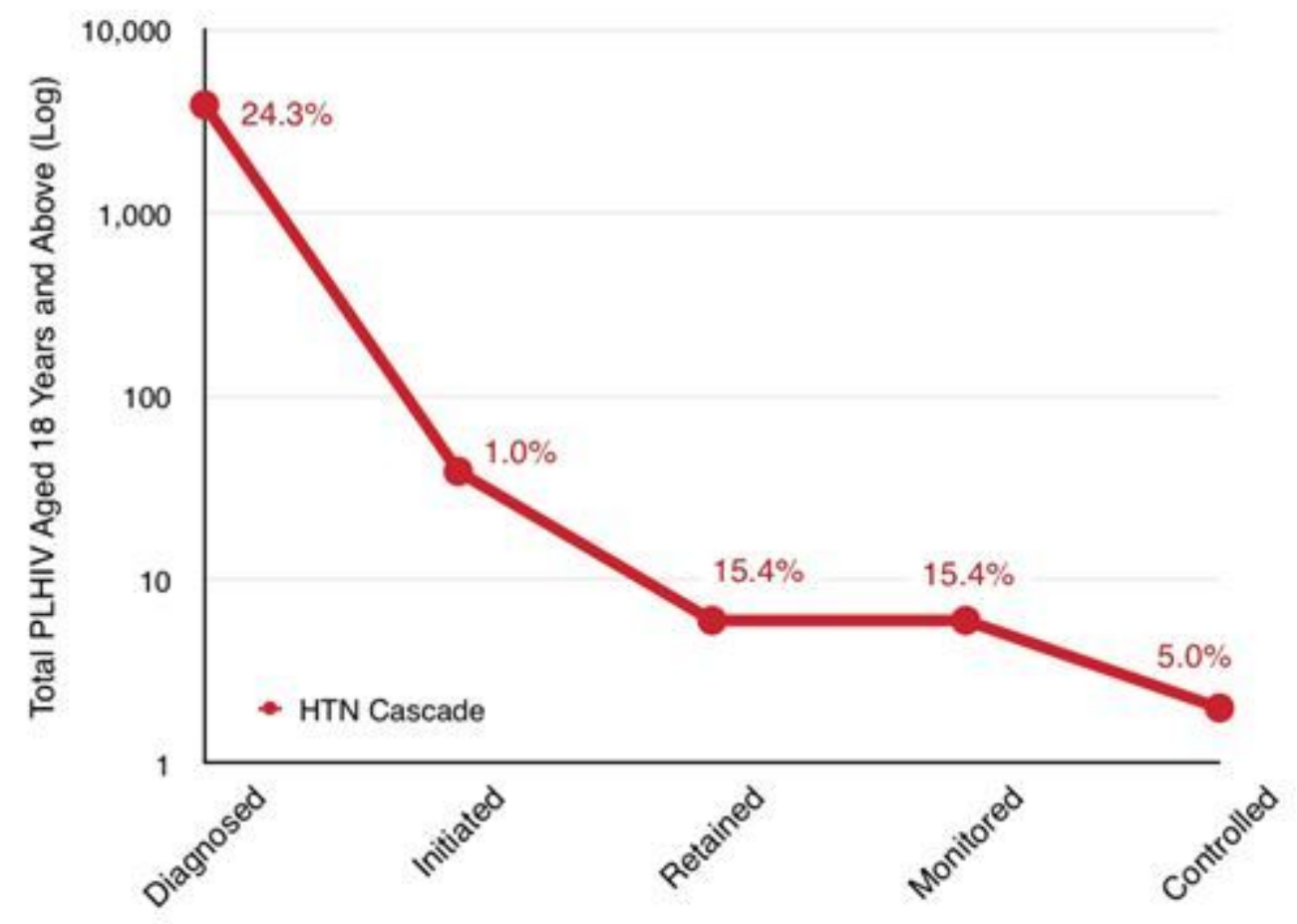

Figure 3

Hypertension care cascade among PLHIV who were diagnosed with HTN at the Mulago ISS clinic The proportions are the percentages of participants at each particular step of the cascade as compared to the previous cascade steps.

\section{Supplementary Files}

This is a list of supplementary files associated with this preprint. Click to download.

- COREQchecklist.DOCX 